\title{
Advanced technique to reduce emissions of fine particulate matter using ultrasounds
}

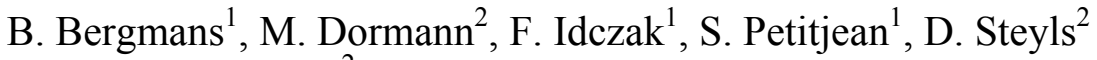 \\ \& B. Vanderheyden ${ }^{2}$ \\ ${ }^{1}$ Institut Scientifique de Service Public (ISSeP), Belgium \\ ${ }^{2}$ Centre for Research in Metallurgy (CRM), Belgium
}

\begin{abstract}
Ever stricter air quality standards force all activity sectors to take measures to reduce the emission of particles. In the past, dust measurements at stack mostly referred to Total Suspended Particles (TSP), but nowadays there are more and more concerns about the finest fractions. Indeed, when inhaled, small particles can go deeply into the lungs and are thus the most toxic ones. Particulate Matters (PMs) are generally classified in different fractions regarding their size $\left(\mathrm{PM}_{10} / \mathrm{PM}_{2.5} / \mathrm{PM}_{1} / \mathrm{UFP}\right)$. A new European standard (ISO 23210) dealing with PM measurements in stacks has been released in 2009 and abatement impositions are expected within short delays. In the framework of various research projects the Centre for Research in Metallurgy (CRM) and its partners are studying the possibility to comply with stricter PM regulation by improving the collection efficiency of existing dedusting systems, in order to avoid expensive investments in additional abatement means. One idea is to agglomerate fine dust particles together or onto coarser ones by applying ultrasounds to the dust laden waste gas flow, upstream of the dedusting unit. This should facilitate the fine dust collection, whatever the considered dedusting technique. The feasibility of this concept has been studied at laboratory scale on a specific pilot for different industrial processes. Specific measurement techniques have also been developed to measure in real time the effects of ultrasounds on the various size fractions. For the moment the abatement reaches already up to $40 \%$ for the smallest particles $\left(\mathrm{PM}_{2.5}\right.$ and $\left.\mathrm{PM}_{1}\right)$, making this system very attractive. Optimization of the method is still under progress. Present activities deal with ultrasounds frequency, acoustic chamber design and up-scaling modelling.

Keywords: ultrasounds, abatement, particles, PM, pre-treatment.
\end{abstract}




\section{Introduction}

PM (Particulate matter) emitted by human activities are responsible for many respiratory diseases in Europe and other industrial countries. Reductions in statistical mean life expectancy that can be attributed to the contribution of fine PM are estimated at 8 months for the European population. The figure for Belgium is one of the highest ones in all European countries with 14 months and reaches up to 2 years in some highly polluted areas [1].

PM10 emissions from large industrial plants should already be reported to fulfil with the European IPPC directive [2]. Impositions on smaller fractions (PM2.5, PM1, and UFP) are also under discussion. A new European standard dealing with PM measurements in stack has been released in 2009 [3]. Measurement campaigns will thus be imposed by environmental agencies and abatement impositions are expected within short delays.

The purpose of this study is to demonstrate the possibility to comply with stricter PM regulations by improving the collection efficiency of existing dedusting systems using ultrasounds technique, in order to avoid expensive investments in additional abatement means.

\section{Material and method}

\subsection{Principle of the concept}

The idea is to agglomerate fine dust particles together or onto coarser ones by applying ultrasounds to the dust laden waste gas flow, upstream of the dedusting unit. This should facilitate the fine dust collection, whatever the considered abatement techniques. Due to their lower inertia, the finest particles are selectively put into vibration by the ultrasonic pressure waves induced by the ultrasounds, resulting in an increase in the number of collisions approving the phenomenon of agglomeration (see figure 1).

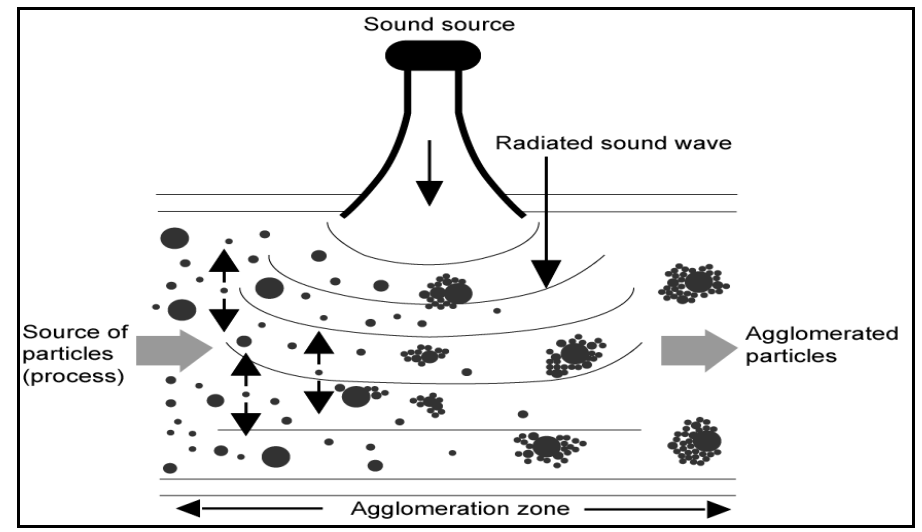

Figure 1: $\quad$ Principle of in-duct acoustic agglomeration of fine dust. 
According to a former study carried out on coal combustion fumes by CSIC [4], PM abatement improvements of $40 \%$ (in mass) can be expected.

\subsection{Description of the pilot installation}

A specific pilot installation (see figure 2) has been built to allow tests on artificial fumes with predetermined composition and concentration of dust. Specific attention has been put in order to obtain regular and homogeneous dust dispersion. Gas composition, flow rate, temperature and moisture can also be controlled to be as close as possible to real plant conditions.

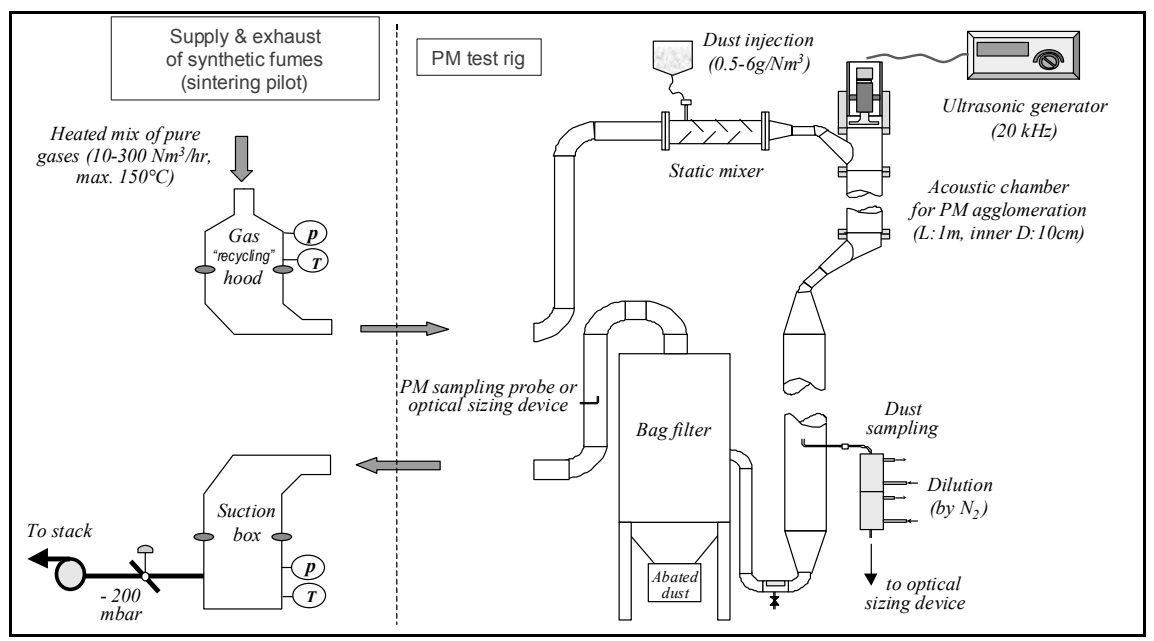

Figure 2: Diagram of the fine dust agglomeration test rig.

The agglomeration chamber is a 1 metre long tube (inner diameter $10 \mathrm{~cm}$ ) with a $20 \mathrm{kHz}$ ultrasounds (US) generator and transducer (sonotrode). The maximum acoustic power achieved is $165 \mathrm{~dB}$. The only material suitable for the ultrasounds emitter in this range of frequencies and powers is titanium, due to its low weight on resistance ratio and its rigidity. A bag filter is used to check the induced dust abatement improvement.

\subsection{Description of the measurement method}

Real time monitoring of particles is necessary to demonstrate the effect of ultrasounds on dust agglomeration. This measurement has been performed using a quantitative dilution system (ejector principle) linked to an optical sizing device (measuring range: from 0.3 to $20 \mu \mathrm{m}$ ). A field oriented prototype has been designed, taking usual constraints of emission measurements and secondary particles formation optimization into account. The validation of the method has been performed in a previous study [5]. 
In order to assess the effect of dust agglomeration on bag filter yield, upstream and downstream measurements were performed simultaneously. Dilution with a factor of 100 is required upstream to avoid the coincidence error (in the optical sizer) due to the high dust concentration. After the bag filter, the use of a diluter is not necessary because the dust concentration is much lower.

Dust collections on Teflon ${ }^{\circledR}$ filters (for weighing analysis and microscope observation) have also been performed to confirm the result of the optical measurements.

\section{Results and discussion}

\subsection{Effect of ultrasounds acoustic power on agglomeration yield}

Figure 3 shows the effect of acoustic power on dust agglomeration. The range of acoustic power tested varies from 157 to $162 \mathrm{~dB}$. Ultrasounds have a significant effect around $160 \mathrm{~dB}$ which confirms the value expected from literature [6]. Results also confirm that the effect of acoustic power is not proportional, there is a value below which no significant results are obtained, followed by a zone with high efficiency and then only very small gain are obtained when increasing the acoustic power. As acoustic level is directly related to the electrical power consumption this parameter is important from an economic point of view.

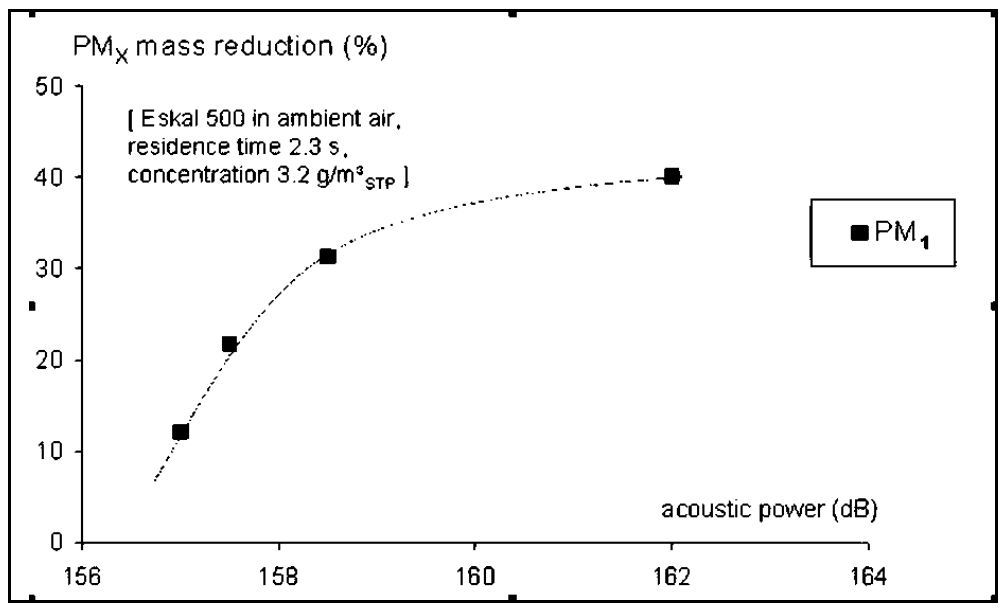

Figure 3: Effect of ultrasounds power source on agglomeration.

\subsection{Effect of residential time in ultrasounds chamber on agglomeration yield}

By increasing the ultrasounds treatment time from 0.6 to 2.3 seconds, a sharp increase of the PM agglomeration efficiency is observed (see figure 4). No significant improvement effect is expected with residence time above 3 seconds. 


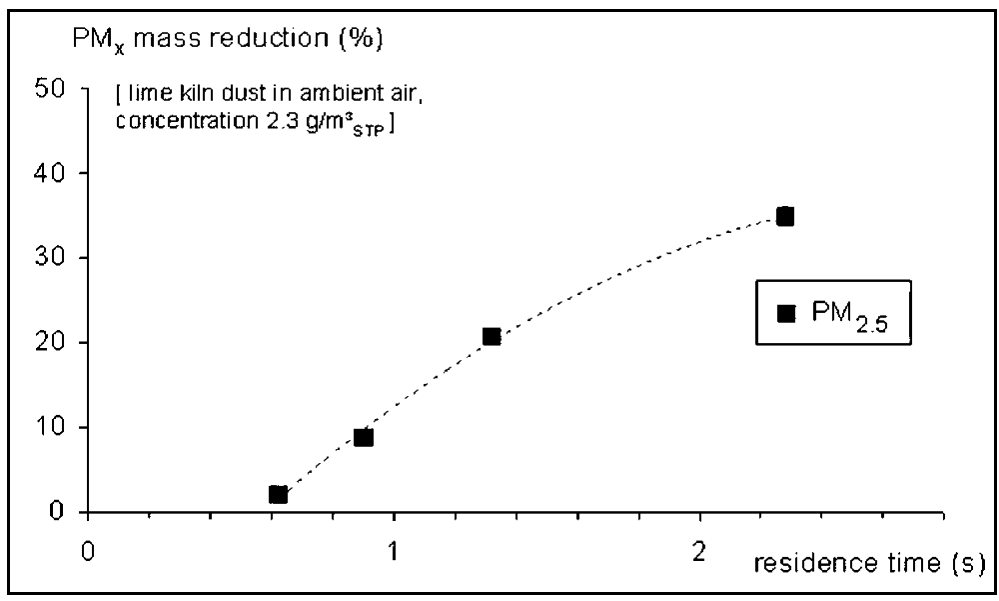

Figure 4: $\quad$ Effect of the residential time on agglomeration.

\subsection{Effect of dust concentration on agglomeration yield}

The effect of dust concentration is presented in Figure 5. A higher concentration in the range of 1.5 to $5 \mathrm{~g} / \mathrm{m}^{3}$ of dust helps to some extent to the particles agglomeration. This phenomenon is simply related to a higher probability of collision at higher concentration.

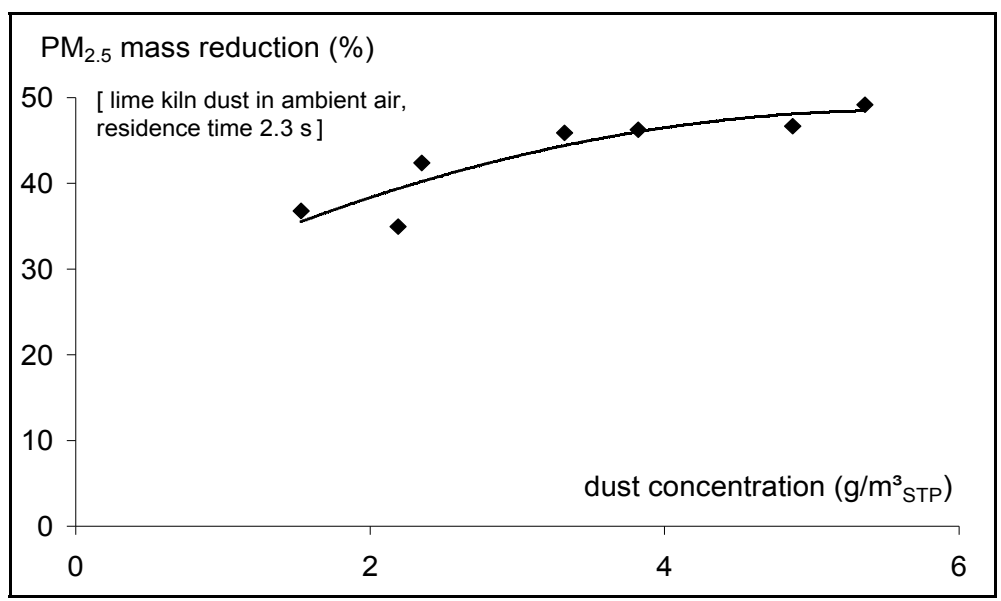

Figure 5: $\quad$ Effect of dust concentration on agglomeration.

\subsection{Effect of ultrasounds on specific class size}

It is interesting to notice that the mass reduction of PM1 is somewhat higher than that of PM2.5 or coarser ones (see Table 1), whatever the acoustic power or 
Table 1: $\quad$ Effect of ultrasounds on dust from different origins.

\begin{tabular}{|c|c|c|c|c|}
\hline & Eskal 500 & ZnO & Lime kiln & Sinter plant \\
\hline Origin & Commercial & Commercial & Sampling & Sampling \\
\hline $\mathrm{v} / \mathrm{v} \%<1 \mu \mathrm{m}$ & 4.5 & 0.7 & 6.0 & 2.1 \\
\hline $\mathrm{v} / \mathrm{v} \%<2.5 \mu \mathrm{m}$ & 20.2 & 4.7 & 11.2 & 14.3 \\
\hline $\mathrm{v} / \mathrm{v} \%<10 \mu \mathrm{m}$ & 97.8 & 22.6 & 53.0 & 17.4 \\
\hline Abatement PM1 & $30 \%$ & $42 \%$ & $26 \%$ & $25 \%$ \\
\hline Abatement PM2.5 & $21 \%$ & $32 \%$ & $20 \%$ & $21 \%$ \\
\hline
\end{tabular}

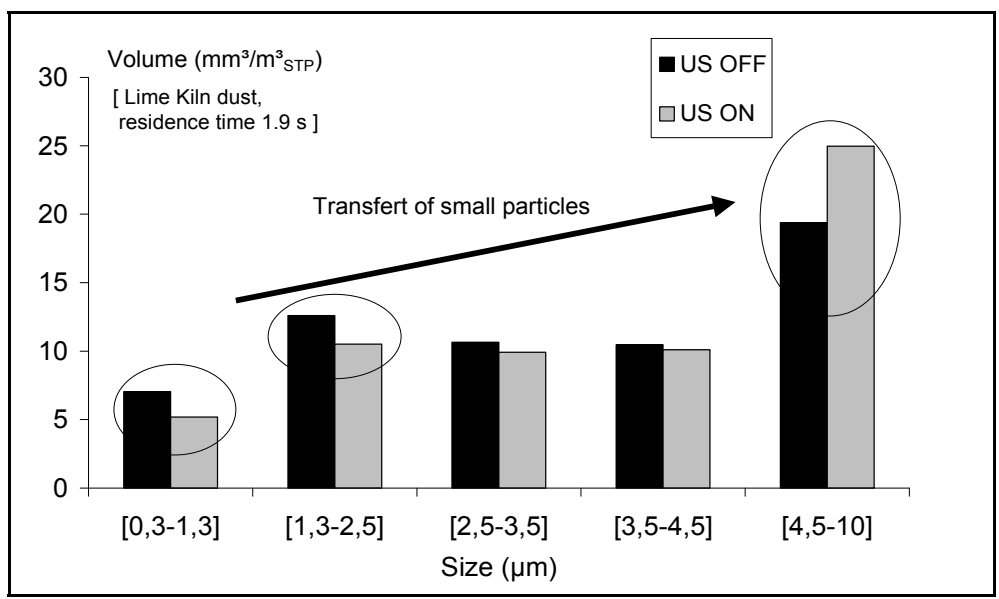

Figure 6: Effect of ultrasound upstream of bag filter.

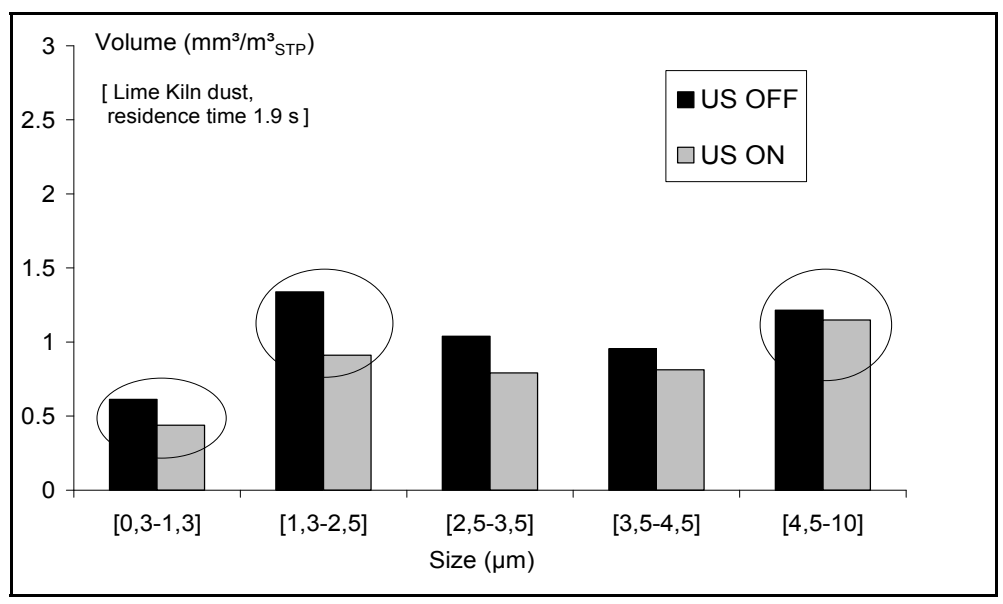

Figure 7: $\quad$ Effect of ultrasounds downstream of bag filter. 
residential time. This effect is due to the high frequency $(20 \mathrm{kHz})$ applied by the sonotrode which selectively puts the finest particles into vibration [7].

Figure 6 shows the variation induced by ultrasounds for five size ranges upstream of the bag filter. One can observe a mass transfer from small particles $(<2.5 \mu \mathrm{m})$ to coarser ones $(>4.5 \mu \mathrm{m})$ when applying ultrasounds.

Figure 7 shows the corresponding results downstream of the bag filter.

The reduction in the smaller size classes is maintained (as a direct result of the preliminary agglomeration of small particles), whereas the increase in the higher size classes is no more observed (as a result of the capture of the clustered particles by the bag filter).

\subsection{Influence of dust type on agglomeration results}

Samples of dust have been collected from various industrial processes or sources to verify the feasibility of this original concept to different industrial processes potentially concerned by the PM issue.

Main dust characteristics and results obtained on them are presented in table 1 .

The first dust (Eskal 500) is a commercial limestone powder used as standard for the calibration of optical sizing systems and made up of hydrophobic, dense (non porous) and nearly spherical limestone particles with a very narrow size distribution $(90 \%$ mass between 1 and $10 \mu \mathrm{m})$. Results are thus expected to be much lower with this technical powder. The three other dusts are industrial flying dusts, collected in existing dedusting systems of a plant. They have wider size distributions with particles up to 100 microns in some cases and 5 to $15 \%$ in mass below 2.5 microns. One has been sampled in steel industry sector (Sinter plant), another one in a zinc oxide production plant $(\mathrm{ZnO})$, and the last one comes from a plant producing cement (Lime kiln).

From these results we can conclude that the PM agglomeration by ultrasounds is a rather universal technique as it is able to significantly reduce the number of small particles whatever the type of dust present in the fume. Contrary to what has been expected, results remain also correct for the technical Eskal 500 powder.

\subsection{Confirmation of the results using the normative measuring technique}

In order to quantify the total suspended dust abatement downstream of the bag house filter, several weighing analysis of samplings collected on filters have been carried out. All sampling are performed in accordance with the requirements of the EN 13284-1 by an accredited laboratory.

Efficiency of a bag house filter increases in time, because the collected dust has also a filtering effect. To avoid an incorrect interpretation of the results, different sequences with and without ultrasounds have been sampled.

Results obtained are presented in table 2 and show a yield in mass abatement around $50 \%$ after the bag house filter when applying ultrasounds to the dust laden waste gas. 
Table 2: $\quad$ Effect of ultrasounds on bag filter yield.

\begin{tabular}{|c|c|c|}
\hline Sequence & US & Dust concentration $\left(\mathrm{mg} / \mathrm{Nm}^{3}\right)$ \\
\hline 1 & OFF & 21.8 \\
\hline 2 & ON & 9 \\
\hline 3 & OFF & 23.2 \\
\hline 4 & ON & 10.1 \\
\hline 5 & OFF & 18.5 \\
\hline
\end{tabular}

These tests have been performed with sinter plant dust. All parameters have been optimized (Residential time $=5 \mathrm{~s}$, maximum ultrasounds power, concentration of dust $=15 \mathrm{~g} / \mathrm{Nm}^{3}$ ). For economical or practical reasons, these parameters can not be implemented in industrial conditions, and abatement around $20-25 \%$ is thus expected in real conditions (see Table 1). Optimization of the technique should still be done before any real field application.

\subsection{Confirmation of the results by microscopy}

Analyses using a scanning electron microscope (SEM) were performed on dust sampled on Teflon ${ }^{\circledR}$ filters to allow a visualization of the agglomeration process.

Figure 6 shows very well the agglomeration of small particles onto coarse ones. The first picture shows a particle collected when no ultrasounds are applied on the fume and the second one the same dust after an ultrasounds treatment.
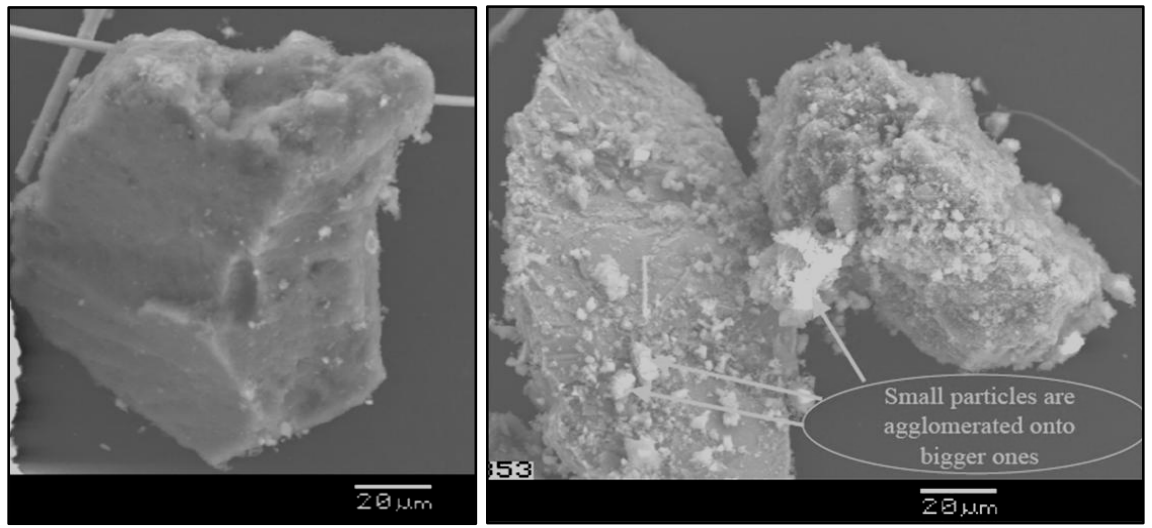

Figure 8: Visualization of the agglomeration due to ultrasounds.

\section{Conclusions}

The improvement of PM abatement on existing dedusting systems can be achieved by an adequate preconditioning of the fumes using acoustic technique. The obtained results show an abatement of up to $50 \%$ and the technique can be applied to a wide range of dust types and operating conditions. 
Source generator and protocol have been optimized on a specific pilot installation and the different parameters (acoustic power, building material, residential time ...) are under control.

Ultrasounds are mainly acting on the smallest particles, which makes this technique very innovating. Other abatement techniques (filters, electrostatic precipitator) remove mainly the coarse fraction and are more or less inefficient on the smallest fractions.

Higher abatement yield could thus be expected on toxic species like heavy metals and Persistent Organic Pollutants (POPs), mostly concentrated on the finest particles. There could be a potentially increased interest for this technique with respect to more health oriented present and future environmental regulations. Abatement of ultra fine particles (UFP) will also be quantified in a near future.

Another advantage is that no substance is injected into the gas contrary to most preconditioning techniques based on additives, so avoiding the related negative side effects (emission or disposal issues).

Future trials aim also to check the influence of gas moisture on PM agglomeration. Indeed the presence of moisture could induce condensation phenomena on the particles modifying favourably their surface properties in terms of agglomeration efficiency and higher agglomeration in the submicron range is expected according to literature [8].

The scaling-up of this technology at industrial level requires the optimization of the acoustic chamber design in order to minimize the number of acoustic generators (for economical reasons) and the residence time (for practical reasons). Within the framework of a second research program, a new acoustic test rig able to work at higher flow rate $\left(200 \mathrm{Nm}^{3} / \mathrm{h}\right)$ and a dedicated simulation tool are for the moment in preparation [9].

\section{Acknowledgements}

This research work is carried out with the financial support of Euregio MeuseRhin (Interreg III) and of the Walloon Region.

\section{References}

[1] Baseline scenarios for the clean air for Europe (CAFE) programme - Final report October 2004

[2] European directive 96/61/CE of 24/09/1996 (JOCE of 10 October 1996) concerning Integrated Pollution Prevention and Control (IPPC) and its implementation decision N(2000) 20004 - 17/07/2000 (JOCE 28 July 2000).

[3] ISO 23210:2009 Stationary source emissions - Determination of PM10/PM2.5 mass concentration in flue gas - Measurement at low concentrations by use of impactors

[4] Gallego et al., Acoustic preconditioning of coal combustion fumes for enhancement of electrostatic precipitator performance, Coal Science 1995, p. $1899-1906$ 
[5] B. Bergmans, F. Idczak, Ph. Maetz and S. Petitjean., Real time monitoring of primary and secondary particulate matter emissions from industrial sources, $8^{\text {th }}$ International Conference CEM $2007-$ III $-45-50-2007$

[6] Shaw, Acoustic particle agglomeration due to hydrodynamic interactions between monodisperse aerosols, Journ. A. Sc. 1979, p. 317-328

[7] Sergey V. Komarov et al., Numerical simulation of acoustic agglomeration of dust particles in high temperature exhaust gas, second international conference on mathematical modeling and computer simulation of metal technologies, 2002

[8] E. Riera-Franco de Sarabia et al., Investigation of the influence of humidity on the ultrasonic agglomeration of submicron particles in diesel exhausts, Ultrasonics 2003, p. 277-281

[9] Paragglo: Reduction of industrial dust pollution by acoustic agglomeration helped by chemical agglomeration, Research project RW DGO6, 2009-2011 